\title{
Systematic Literature Review Update of the PROUD Trial: Potential Usefulness of a Collaborative Database
}

\author{
Frederic C. Daoud
}

To the Editor:

$\mathbf{T}$ HE PROUD TRIAL, which is registered in Germany and is examining the effects of suture type on surgical site infection (SSI), was published shortly before our systematic literature review $[1,2]$. The methods met the eligibility criteria of our review protocol. An extraction of literature databases returned no other new relevant study. The metaanalysis was updated with the 16th trial (Fig. 1). It presented a relative weight of $17.1 \%$ and did not introduce significant publication bias (Egger regression $\mathrm{p}=0.085$ ) or heterogeneity ( $\mathrm{Q}$-test $\mathrm{p}=0.112$ ). The results were consistent with those of the 15-trial meta-analysis. The randomeffects risk ratio (RR) was 0.71 with a $95 \%$ confidence interval (CI) of 0.58-0.87 $(\mathrm{p}<0.0012)$. Sensitivity analysis confirmed the robustness of the results. The three trials with the highest relative weight after the PROUD trials had to be removed jointly for the pooled RR to become nonsignificant: $0.79,95 \%$ CI $0.79-1.01, \mathrm{p}<0.057$ [3-5]. That trial considerably strengthened the polydioxanone subgroup but had little effect on the results of covariable analysis.

The data that could be pooled from the 16 trials so far did not enable an explanation of the large variations in RR across trials. Moreover, publications did not describe the treatments and outcomes of the SSIs systematically, so the impact of suture type on burden-of-illness could not be estimated.

Leveraging source data of current trials could be attempted through a collaborative database of individual data that should include treatments and outcomes of SSIs that could be retrieved from patient charts. Individual level meta-analysis would increase the power to detect covariables that explain the large differences in RRs across trials and would also enable estimation of burden-of-illness.

If this approach proves to yield more knowledge from current trials, ongoing and future trials could consider participating in this collaborative effort in order to leverage the contribution of their own findings to the overall evidence.

Random-effects pooled RR of SSI - 16 RCTs

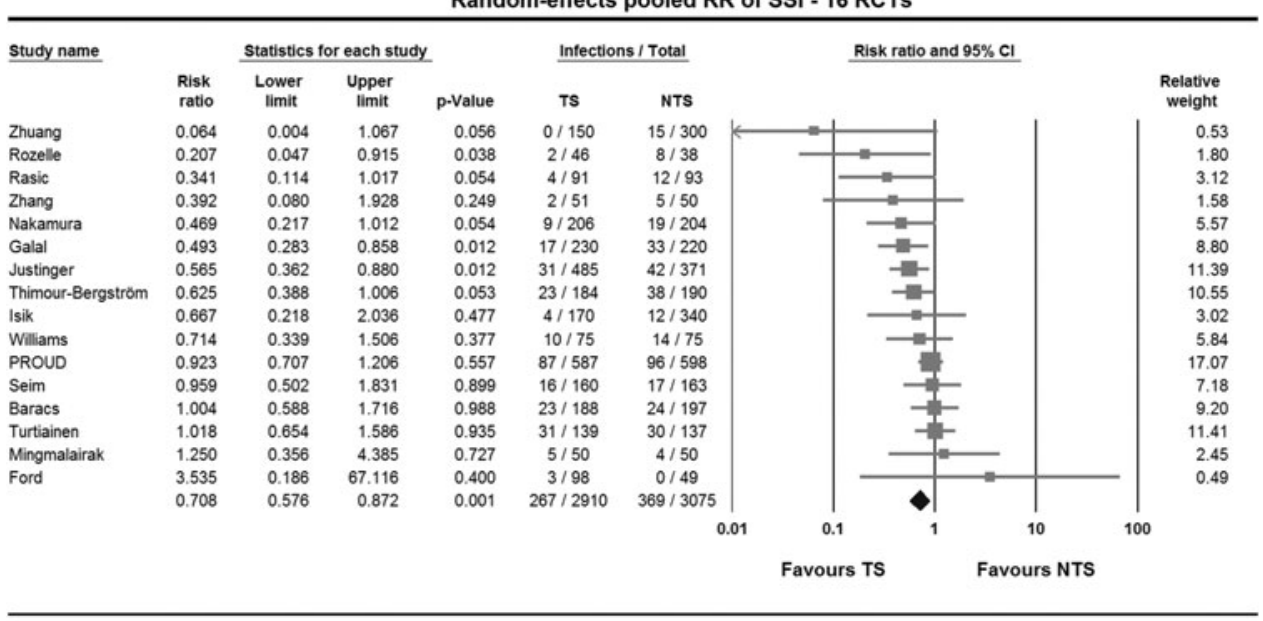

RR: Risk Ratio, SSI: Surgical Site Infection, TS: Triclosan Suture, NTS: Non-Triclosan Suture, RCT: Randomized Controlled Trial

FIG. 1. Random-effects pooled relative risk of surgical site infection in 16 randomized controlled trials of different suture materials.

Unit of Biostatistics \& Bioinformatics, Medextens, Paris, France. 


\section{Author Disclosure Statement}

This letter is the sole responsibility of the author. No external funding or other form of support was received from non-profit or for-profit entities.

\section{References}

1. Daoud FC, Edmiston CE Jr, Leaper D. Meta-analysis of prevention of surgical site infections following incision closure with triclosan-coated sutures: Robustness to new evidence. Surg Infect 2014;15:165-181.

2. Diener MK, Knebel P, Kieser M, et al. Effectiveness of triclosan-coated PDS Plus versus uncoated PDS II sutures for prevention of surgical site infection after abdominal wall closure: The randomised controlled PROUD trial. Lancet 2014 Apr 4. DOI: 10.1016/S0140-6736(14)60238-5 [Epub ahead of print].

3. Galal I, El-Hindawy K. Impact of using triclosan-antibacterial sutures on incidence of surgical site infection. Am J Surg 2011;202:133-138.
4. Thimour-Bergström L, Roman-Emanuel C, Scherstén H, et al. Triclosan-coated sutures reduce surgical site infection after open vein harvesting in coronary artery bypass grafting patients: A randomized controlled trial. Eur J Cardiothorac Surg 2013;44:931-938.

5. Justinger C, Slotta JE, Ningel S, et al. Surgical-site infection after abdominal wall closure with triclosan-impregnated poliydioxanone sutures: Results of a randomized clinical pathway facilitated trial (NCT00998907). Surgery 2013;154: 589-595.

Address correspondence to: Dr. Frederic C. Daoud MEDEXTENS SARL 75 rue de Lourmel 75015, Paris, France

E-mail: fcdaoud@medextens.com 